\title{
Human papillomavirus vaccine coverage among the intellectually and developmentally disabled: A single-institution study.
}

\author{
Kim HG ${ }^{1 *}$, Ancar FM ${ }^{1,2}$, Kauffman RP ${ }^{1,2}$, Baker $T^{1,2}$ \\ ${ }^{1}$ Texas Tech University Health Sciences Center School of Medicine, Amarillo, TX, USA \\ ${ }^{2}$ Department of Obstetrics and Gynecology, Texas Tech University Health Sciences Center, Amarillo, TX, USA
}

\begin{abstract}
Objective: Human papillomavirus (HPV), the most common sexually transmitted infection, can cause genital warts and cancers. While HPV vaccines can protect against these diseases, the intellectually and developmentally disabled population may not be adequately receiving this routine health care.

Methods: Patients, between age 9 and 26, seen at a rural academic institution from January 2013 to December 2016 were included. Patients with intellectual disability and developmental delay and those who received HPV vaccines were searched using medical codes. Vaccination rates by age, sex, and department were analyzed.

Results: HPV vaccination rates of the disabled and non-disabled patients were $18.4 \%$ and $17.7 \%$, respectively. Among the disabled, younger aged and female patients, seen at the Department of Pediatrics, were vaccinated more often.

Conclusions: While the overall HPV vaccination rate was low, there was no significant difference between the disabled and non-disabled, suggesting that all patients, regardless of disability, are receiving the equal level of care.
\end{abstract}

Keywords: Human papillomavirus, Vaccination, Intellectual disability, Developmental disability, Cancer prevention, Immunization.

Accepted on June 20, 2018

\section{Introduction}

Human papillomavirus (HPV) is the most common sexually transmitted infection (STI) in the U.S. 79 million Americans, most in their late teens and early $20 \mathrm{~s}$, are infected with the virus. While most HPV infections are asymptomatic, they can result in various health problems. Non-oncogenic types, such as types 6 and 11, can cause anogenital warts. Oncogenic types, such as types 16 and 18 , can cause cervical and other cancers, including cancer of the vulva, vagina, penis, anus, and oropharynx. Nevertheless, vaccination against HPV can prevent these diseases. Cervarix protects against types 16 and 18, Gardasil against 6, 11, 16, and 18, and Gardasil 9 against five additional types-31, 33, 45, 52, and 58. They are available for both males and females from ages 9 to 26 [1].

Although HPV vaccine has become a routine intervention given to adolescents to prevent various cancers, this standard of care can be undermined in the medically underserved and minority populations, including the intellectually and developmentally disabled (IDD) [2,3]. We hypothesized that IDD patients may not be receiving the vaccine as consistently as their non-disabled peers. Therefore, we compared the rate of HPV vaccination in the IDD population to the non-disabled population at a large rural academic institution.

\section{Materials and Methods}

The study was approved by the Institutional Review Board of the Texas Tech University Health Sciences Center (TTUHSC). This retrospective review included patients, aged 9 to 26, seen at Departments of Family Medicine, Pediatrics, Internal Medicine, and Obstetrics and Gynecology clinics at TTUHSC between January 1, 2013 and December 31, 2016.

Among those patients, individuals with mental retardation, intellectual disability, or cognitive and/or developmental delay were separated by searching International Classification of Diagnoses (ICD)-9 and ICD-10 codes ("IDD"). A similar number of patients without the diagnoses were randomly selected ("Control"). From each group, the number of individuals who received the HPV vaccination was determined by searching ICD-9, ICD-10, and Current Procedural Terminology codes.

Vaccination rates by age, sex, and department were analyzed. The Chi-square test of association was performed using Med Calc 18.2.1, Ostend, Belgium, and a $\mathrm{P}$ value of $<0.05$ was statistically significant.

\section{Result}

Among the 15,305 patients who met the criteria, a total of 341 patients fell into the IDD group, and 350 out of 14,964 patients were randomly selected to the control group.

The number of intellectually disabled and non-disabled patients who received HPV vaccination was 63/341 (18.4\%) and 62/350 $(17.7 \%)$, respectively $(\mathrm{p}=0.80)$ (Table 1$)$. 
Citation: Kim HG, Ancar FM, Kauffman RP, et al. Human papillomavirus vaccine coverage among the intellectually and developmentally disabled: a single-institution study. J Child Adolesc Health. 2018;2(2):1-24.

Table 1. HPV vaccine coverage in the non-disabled versus intellectually and developmentally disabled population.

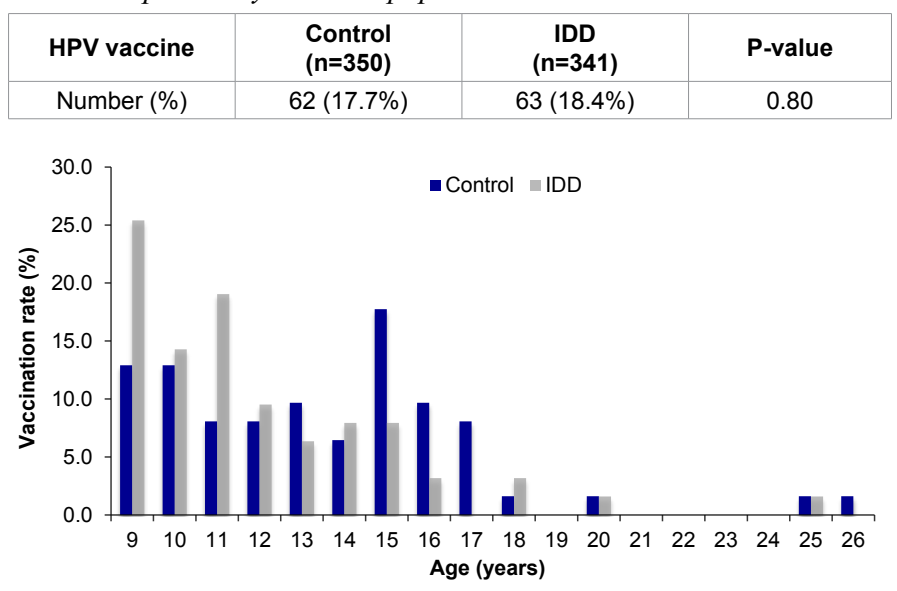

Figure 1. HPV vaccination rates in the non-disabled and intellectually and developmentally disabled population by age.

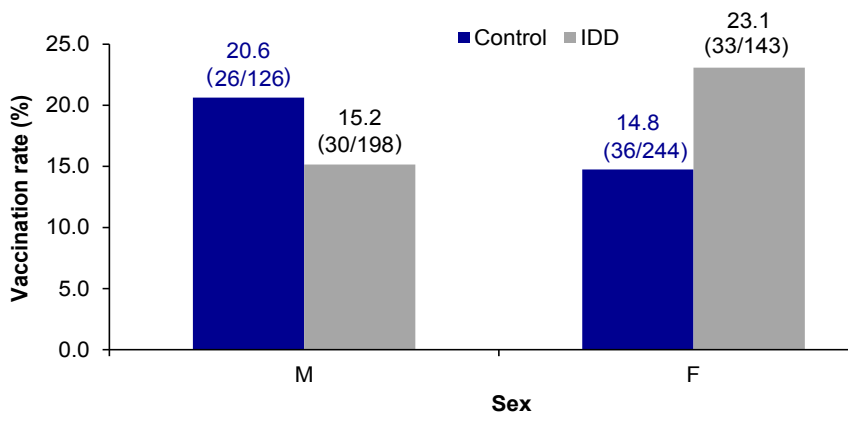

Figure 2. HPV vaccination rates in the non-disabled and intellectually and developmentally disabled population by sex.

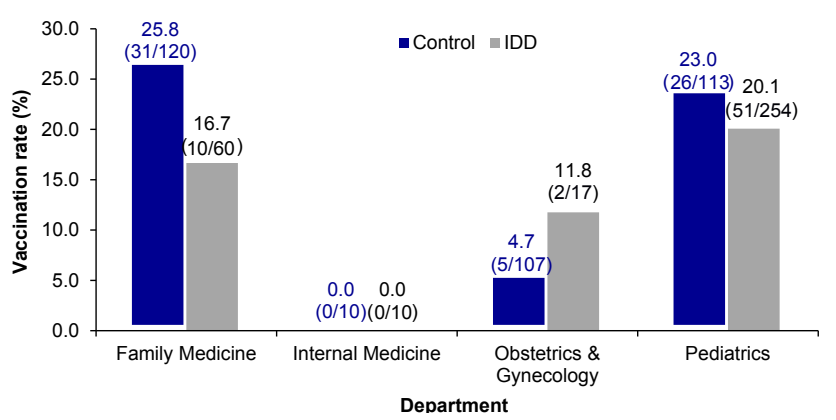

Figure 3. HPV vaccination rates in the non-disabled and intellectually and developmentally disabled population by department.

The vaccination rates among the IDD versus control groups varied depending on the patient's age, sex, and department where they were seen. The control group showed a bimodal distribution while the IDD group had a decreasing trend as the patient age increased (Figure 1). Males in the control and females in the IDD group had higher vaccination rate (Figure 2). The Department of Family Medicine in the control and Pediatrics in the IDD group had the highest rate (Figure 3).

\section{Discussion and conclusion}

HPV infection is more prevalent among minorities and the medically underserved, including the IDD [2,3]. Gynecologic care for women with these disabilities poses unique challenges. Not only taking a gynecologic history, including menstrual and sexual history, but also physical examinations in these patients can be complicated. They also may not fully comprehend or be able to consent to certain invasive procedures [4].

IDD patients are often perceived as asexual or childlike by health care providers and their caregivers. However, this perception is often incorrect; it is well-documented that sexual activity in the IDD population is indeed an expression of sexuality and a common behavior. In fact, the literature shows that women with mild to moderate intellectual disability are more likely to engage in sexual activity. Nevertheless, many of these sexual relationships occur secretly, posing a greater risk of spread of and exposure to STI $[5,6]$.

Societal assumptions can lead to parents' assumption that their intellectually disabled teenager or adult is sexually inactive and would have no need for the HPV vaccine. Therefore, it is crucial for providers to offer and counsel about the HPV vaccine to all individuals who meet criteria for its administration.

While the overall HPV vaccination rate was low at our institution, there was no significant difference between the rates in disabled and non-disabled patients. This suggests that providers are delivering the same level of care to all patients, regardless of disability. Among the IDD, younger aged and female patients, seen at the Pediatrics clinic, were vaccinated more often.

One limitation of this study is that only the HPV vaccination during patients' specific visits at a single institution was reviewed. While vaccines are usually given by primary care physicians, patients visit our clinics more for specialist care, which could account for the low rate. Moreover, the patients' vaccination status prior to and post-visit at our clinics is unknown. Further studies are necessary to examine if this low rate is due to the inadequacy of providers' role in counseling or the patients' choice to opt out.

\section{Acknowledgement}

The authors wish to acknowledge the contribution of the Texas Tech University Health Sciences Center Departments of Internal Medicine, Family Medicine, Pediatrics, and Obstetrics and Gynecology for their assistance with this research.

\section{References}

1. HPV. HPV Vaccine information for clinicians: Fact sheet. 2011;2:1.

2. Perkins RB, Brogly SB, Adams WG, et al. Correlates of human papillomavirus vaccination rates in low-income, minority adolescents: A multicentre study. J Women Health. 2012;21(8):813-20.

3. Mac Leod R, Tuffrey C. Immunisation against HPV in girls with intellectual disabilities. Arch Dis Child. 2014;99(12):1068-70.

4. Kavoussi SK, Smith YR, Ernst SD, et al. Cervical cancer screening with liquid cytology in women with developmental disabilities. J Women Health. 2009;18(1):115-8.

5. Eastgate G, Van Driel ML, Lennox N, et al. Women with 
intellectual disabilities: A study of sexuality, sexual abuse and protection skills. Aust Fam Physician. 2011;40(4):226-30.

6. Jaffe JS, Timell AM, Eisenberg MS, et al. Low prevalence of abnormal cervical cytology in an institutionalized population with intellectual disability. J Intell Disabil Res. 2002;46(7):569-74.

\section{*Correspondence to:}

Hyunyoung G Kim

Texas Tech University Health Sciences Center

School of Medicine

Amarillo, TX 79106

USA

Tel: +1 (806) 414-9650

E-mail: hyunyoung.kim@ttuhsc.edu 